\title{
Appareil
}

11 | 2013

L'espace et l'architecture: état des lieux

\section{À travers les villes : Europe/USA/Antonioni, une hétérotopie en mouvement}

Jean-François Robic

\section{OpenEdition}

\section{Journals}

Édition électronique

URL : http://journals.openedition.org/appareil/1789

DOI : 10.4000/appareil. 1789

ISSN : 2101-0714

Éditeur

MSH Paris Nord

Référence électronique

Jean-François Robic, «À travers les villes : Europe/USA/Antonioni, une hétérotopie en mouvement », Appareil [En ligne], 11 | 2013, mis en ligne le 26 septembre 2013, consulté le 30 juillet 2020. URL: http://journals.openedition.org/appareil/1789; DOI : https://doi.org/10.4000/appareil.1789

Ce document a été généré automatiquement le 30 juillet 2020.

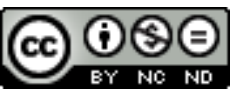

Appareil est mis à disposition selon les termes de la Licence Creative Commons Attribution - Pas d'Utilisation Commerciale - Pas de Modification 4.0 International. 


\title{
À travers les villes : Europe/USA/ Antonioni, une hétérotopie en mouvement
}

\author{
Jean-François Robic
}

\section{NOTE DE L'AUTEUR}

Contrairement aux habitudes d'écriture, nous avons choisi de restituer au film son titre tel qu'il apparaît aux génériques d'ouverture et de fin, sans le tiret qui relie les deux mots. Néanmoins, lorsque ce titre apparaît dans celui d'un ouvrage ou d'un article, ou dans une citation, nous conserverons l'orthographe fautive habituellement acceptée.

1 Aucune ville n'est homogène. Elles se sont construites différemment, dans l'histoire, dans l'espace. Elles présentent toutes ou presque une collection de formes qui finissent toujours quelque part par faire une collection d'images, une sorte de Mnémosyne urbaine de laquelle nous pouvons mettre en lien les différents points, qui sont toujours des points de vue... Traverser ces villes constituait autrefois une manière de les mesurer. Mais les traverser comment? Une façon de traverser les villes serait de le faire par le biais des images, puisque nous parlons de points de vue. Je ne dis pas «en image ", mais bien "par le biais", c'est-à-dire qu'il s'agirait d'observer comment la ville et les images se construisent ensemble, dans le même mouvement. Walter Benjamin souligne bien ce mouvement dans Paris, capitale du XIX ${ }^{e}$ siècle $^{1}$ : Haussmann construit sa ville comme s'il s'agissait d'une image en perspective, et tout le livre soustend la connivence entre la photographie, l'urbanisme et l'architecture, tous fondés sur la mise en perspective. Urbanisme et images, le cinéma peut nous aider à comprendre cela d'une autre manière, puisque l'art du mouvement peut restituer dans le temps et dans l'espace la traversée des apparences urbaines...

2 Autrefois, Paris se traversait en une heure, à pied. Aujourd'hui, on n'imagine même pas pouvoir le faire, faute de temps et faute de sécurité... Même les flâneries les plus 
proches des surréalistes ou des situationnistes nous semblent loin des circonstances actuelles. Les "moyens de transport» sont devenus obligés, avec la modernité industrielle. La voiture même n'est plus le moyen privilégié pour cela, trop individuel, trop polluant, etc. Pourtant, il fut une période où tout l'urbanisme a pu être plié à l'automobile « familiale » ou individuelle, tant celle-ci paraissait à la fois horizon social du plus grand nombre et source de profit des autres... Ce temps n'est pas très éloigné, il n'est peut-être pas fini même s'il est écorné, et du point de vue de l'art, de l'esthétique, cette période, celle des années 1950 à 1970, constitue une référence estimable pour beaucoup d'artistes encore en activité. Pour les philosophes ou les urbanistes, je ne peux pas répondre...

3 La ville de cette époque-là a été un modèle, ou un motif iconographique fort. Je souhaiterais proposer ici l'appropriation de ce motif par l'un des cinéastes les plus représentatifs de cette période, Michelangelo Antonioni. Celui-ci, à travers les films de cette époque, propose une vision urbaine par le motif de la traversée, renforçant la visibilité de l'hétérogénéité par le moyen du déplacement, dans lequel l'automobile - et une forme particulière de celle-ci - devient une machine à voir, voire une sorte d'appareil à condition que l'on n'y voit pas un médium au sens de la médiologie. L'automobile, on va le voir, dépasse largement son statut social et son rôle symbolique (qu'une iconographie pourrait reconnaître), et se justifie totalement cinématographiquement.

4 Certes, il s'agit ici de représentation et de fiction, mais il existe chez Antonioni une acuité dont la source est le fondement documentaire de son œuvre : il commence en effet sa carrière comme documentariste ${ }^{2}$. Il y a donc, je pense, une réelle volonté chez lui de donner cette pensée de la ville qu'il construit visuellement depuis le début de sa carrière soit comme documentariste, soit comme cinéaste de fiction.

5 Trois films le conduisent particulièrement à proposer ces visions urbaines, à travers le déplacement obligé que nécessite l'appréhension de cette hétérogénéité urbaine, et qui se fait dans le temps autant que dans l'espace, selon la nature du cinéma. Le cinéaste italien construit cette vision d'abord dans une forme qui rend compte de la ville à travers un déplacement rapide, elliptique et «descriptif» dans L'Avventura. Progressivement cette vision devient autonome dans Blowup, jusqu'à constituer une esthétique propre, « autonome ", avec Zabriskie Point. Mais cette autonomie quasi-totale qu'elle acquiert dans ce dernier exemple, la forme cinématographique nous fait sentir que cette vision est tributaire de la forme de la ville elle-même et de la manière dont on la traverse. Ce qui forme un indice (au sens déductif) pour penser qu'au bout du compte c'est sans doute à chaque fois une explication valide.

Ainsi dans L'Avventura, Rome se traverse à rebours de sa profondeur historique, d'un lieu actuel, en construction, à un lieu plus ancien, au travers de formes antiques; quelque part, Rome reste une image, collage culturel et hyper-lieu imaginaire. Londres, dans Blowup, est l'archétype de la ville moderne avec ses quartiers divers, alternant un développement ancien horizontal et des quartiers d'affaires verticaux. Il se traverse plusieurs fois dans le film, mais jamais autant avec la logique "serrée ", la cohérence cinématographique de cette séquence particulière. La linéarité du trajet est traduite visuellement mais sur un rythme temporel et visuel qui privilégie la vision par le mouvement cinématographique à l'aide du point de vue embarqué, ici aussi comme dans L'Avventura. Mais cela ne dure pas longtemps: la ville impose au cinéma ses ruptures formelles qui se coulent ici dans le montage cinématographique. Blowup 
propose par ailleurs plusieurs traversées automobiles au travers de quartiers différents dont l'addition cinématographique propose un mélange formel déroutant. D'autre part, comme la Rome de L'Avventura mais différemment, Londres est une ville en train de se faire, il y a des formes en construction sur lesquelles Antonioni s'attarde volontairement; de plus c'est un espace qui se charge de symboles comme on le verra, alors même que la Rome du premier film s'en trouve curieusement démunie, là où l'on s'y serait pourtant attendu. Enfin dans Zabriskie Point, Los Angeles nous apparaît dans une absence d'identité spatiale et historique, dans une isomorphie démesurée, et se traverse "n'importe comment", de façon chaotique et cahotante, encore plus déroutante que précédemment, dans un mouvement constant de la caméra/du regard, à la limite de l'hystérie visuelle et sonore. Car plus que dans les deux autres films, la séquence de la traversée automobile de LA est un moment audio-visuel très marqué et qui commence d'ailleurs - ce n'est pas un accident ou un hasard, - par un travelling le long d'une fresque dont l'auteur s'est tué en la réalisant... Trois films donc, trois traversées, mais trois villes différentes dans leurs structures urbaines et leurs formes plastiques. Et donc trois moments cinématographiques très singuliers portant ces trois visions comme on porte un étendard, c'est-à-dire comme un point focal sur lequel on ne lâche pas le regard pour comprendre où on est. Où : c'est-à-dire dans quelle ville et dans quel film.

Pour en finir avec cette présentation, on croisera ici la notion d'hétérotopie, mais conçue comme une dynamique et non comme un lieu dont la seule profondeur et la singularité en feraient un objet statique ou simplement différentiel, voire un lieu littéralement hors-là. C'est peut-être encore le cas dans ce film totalement hétérotopique qu'est L'Avventura où la traversée se retrouve si coincée entre deux secteurs urbains qu'elle n'a pas le temps d'apparaître comme un lieu elle-même. Mais une dynamique du mouvement cinématographique porte les deux autres traversées vers une forme qui va progressivement, de Blowup à Zabriskie Point, devenir mobile, comme une liaison hyper-hétérotopique. Et l'instrument de cette liaison, c'est la machine à voir automobile.

\section{L'automobile, machine à voir, transport de la vision}

8 Avant d'en venir à la forme de la vision elle-même, parlons de ce qui la permet, son médium : l'automobile. Loin de moi l'idée de construire une médiologie de la voiture, sans doute déjà faite. Plutôt considérer ici ce moyen de transport de la vision comme une machine de vision, un médium donc. Ainsi, ces trois films proposent, en tant qu'iconographie, le déplacement à travers la ville au moyen de l'image mobile de la voiture dans les rues, et cela dès les premières images pour certains, mais encore la voiture porte l'instrument, la caméra embarquée. Plus encore, elle prête sa vision propre au cinéma : on voit la ville depuis la voiture, et on voit la ville comme la voiture la voit - si l'on me pardonne de donner ainsi une conscience perceptive à une machine, mais je ne serai pas le premier. La forme cinématographique de la ville prend naissance dans le mouvement de la voiture et dans sa vitesse, en elle-même variable.

9 La voiture est une sorte de personnage de par le rôle qu'elle joue et à chaque fois de par sa catégorie particulière. En effet, elle n'est pas n'importe quelle voiture: un coupé décapotable, un cabriolet. Dans les films convoqués, ce cabriolet est l'élément permanent. Mis à part Zabriskie Point où elle est, pour la séquence qui nous occupe, une 
camionnette, dans L'Avventura, Blowup, mais aussi dans Profession Reporter (dont je ne parlerai pas), à chaque fois une caméra est embarquée dans un coupé décapotable: quel(s) type(s) de vision permet cette situation scénographique? Quel regard permetelle sur la ville? Le cabriolet n'occulte pas la rue. Il n'est pas un obstacle pour la caméra, et sa vision se mêle avec celle de l'appareil cinématographique, nouvel appareil liant l'univers photo-cinématographique et l'univers automobile, pour des connexions infinies. En général les voitures sont fermées : protection des dangers, de la météo, des regards, isolement dans la ville, ne pas se laisser pénétrer par elle. Ici au contraire le cabriolet offre une ouverture déployée vers le paysage urbain et un renforcement de la mobilité de la caméra qui est ainsi plus libre, proposant également un couplage plus fort du conducteur avec le paysage contre une fermeture protectrice de la berline.

On reconnaîtra peut-être dans mes propos une idée reprise à L'esthétique de la disparition $^{3}$ de Paul Virilio, qui encourage cette analogie entre cinéma et automobile, en développant la similitude entre le pare-brise et l'écran, et le mouvement de la voiture avec celui du cinéma ${ }^{4}$. Cela dit, nous ne sommes pas ici dans le cas d'une berline fermée, formant « salle » obscure où le cinéma se contemple. L'occurrence est différente en ce que la voiture en question est toujours une décapotable : la fermeture a disparu, nous sommes en plein air, dans une sorte de drive-in, cette fois-ci bien nommé... Cela élargit la vision, déborde l'écran, ne met pas d'obstacle à une vision à $360^{\circ}$. Et cela met le conducteur, et ainsi peut-être le spectateur, en danger : trop d'air, trop de bruit, trop de mouvement, et aussi trop d'images... Un effet qui avoisine l'ivresse, celle qui se manifeste dans les situations de déséquilibre, comme de tourner sur soi en vitesse.

11 Ici même, pour faire une digression, notons que les voitures d'Antonioni ne sont pas les mêmes à chaque fois et que si la vision change dans des environnements urbains différents, l'automobile propose aussi de caractériser celui qui la conduit ou se déplace avec. Que représente la décapotable dans l'univers urbain et les représentations de cette époque ? Elle est l'écho de plusieurs mythologies modernistes : reconnaissance et réussite sociale, individualisme (on n'y trimbale pas sa famille), l'élitisme (dans Blowup c'est aussi une Rolls Royce), starlétisation, sport... Dans L'Avventura, le cabriolet Hillman Minx Phase V Convertible 1951 est conduit par un chauffeur, ce qui classe ses passagères, l'une d'entre elles au moins, comme une fille de très bonne famille (le film par ailleurs commence dans une villa chic sur les hauteurs de Rome). Dans Blowup, le photographe n'a pas besoin de chauffeur, c'est un être indépendant, très fortement individualiste, un artiste: mais c'est une Rolls Royce... le dessus du paquet, la Silver Cloud III Mulliner Parkward Drophead Coupé, aka Chinese eyes, un modèle " édité » à 100 exemplaires par le carrossier Mulliner Park Ward Ltd en 1965. À un moment du film, le photographe fait cette remarque qui fait sourire : « Si j'avais de l'argent je serais libre... » Pour Zabriskie Point, il n'y a pas de cabriolet, c'est un autre monde, seulement un vieux camion bringuebalant, un Ford F-Series de 1963... Mais c'est justement ce bringuebalement qui va à son tour "produire» la sorte de vision - voire l'audiovision - de la ville américaine telle qu'Antonioni nous la propose : tout comme le cabriolet, la camionnette est à son tour une machine à voir. En effet, plusieurs plans sont d'ailleurs filmés depuis le plateau du pick-up : la configuration de cette voiture propose une position ambivalente permettant à la fois de visualiser le paysage depuis la voiture et de porter le regard de la caméra vers l'intérieur de l'habitacle.

12 Quoi qu'il en soit, Antonioni n'est pas le seul à cette époque, à utiliser ce dispositif cabriolet + caméra : Godard le fait dans Pierrot le fou (1965), Jacques Demy avec son film 
américain Model Shop (1969), qui marche à la fois sur les pas de Blowup et prévisualise Zabriskie Point. Et bien d'autres sans doute, tel Stanley Kramer pour le dernier plan du Dernier rivage (On the Beach, 1959)... La voiture décapotable est-elle un nouveau médium qui remplace ou constitue un dispositif technique appareillant la vision dans sa conjonction avec la caméra cinématographique, déjà en œuvre et explicitement montré dans L'Homme à la caméra de Vertov ? Cet exemple, étant un film sur la poïétique du film lui-même, montre assez clairement que dès le début du cinéma "mobile " l'alliance caméra-décapotable semble tentante pour les cinéastes qui souhaitent produire une vision dynamique et animée de la ville et du paysage. En tout cas, l'objet lui-même est récurrent dans toute l'histoire du cinéma, il forme une sorte de mythologie qui va de Dziga Vertov et Buster Keaton ${ }^{5}$ à David Lynch (Sailor et Lula, Blue Velvet), en passant par Hitchcock qui en use et abuse, Godard évidemment - qui en fait aussi une machine de mort à la fin du Mépris -, et bien sûr les James Bond. Gageons aussi que le véhicule de Luke Skywalker, dans Star Wars IV, ressortit du genre. Réservons notre attention à ce monde en soi que constituent les années 1960, sans doute parce que si Lynch, par exemple, ou Tarentino encore, l'utilisent tant c'est sans doute en raison de sa présence dans le cinéma moderne des années pop... À tout seigneur tout honneur.

\section{Le déplacement, une forme de l'hétérotopie : le cas de Blowup}

13 Par le biais du cabriolet, le déplacement témoigne de la ville, qu'il soit l'expérience réelle pour tout un chacun ou qu'on l'envisage dans sa traduction cinématographique. Il faut considérer le déplacement comme une forme de la ville, au même titre que l'architecture, l'infrastructure des services ou les rapports sociaux qui en découlent. Par conséquent, le déplacement induit aussi des changements dans la vision traditionnelle en fonction de son évolution technique et permet de comprendre et renouveler le mouvement de la ville. Il permet aussi d'en apprécier la diversité, son hétérogénéité spatiale et formelle. Dans la séquence de Blowup où l'on voit pour la première fois le photographe se rendre en direction du parc, la ville change au fur et à mesure que la voiture se déplace. C'est déjà le cas dans la séquence initiale de L'Avventura, où l'on passe en voiture d'une typologie urbaine à une autre, mais Blowup radicalise ce propos et permet d'en montrer plus dans une séquence plus longue. Ainsi le film change en fonction du déplacement: d'une part, c'est ce déplacement qui est le véritable début de l'intrigue, après 20 minutes d'une chronique ordinaire, ensuite, de tous les déplacements montrés dans le film, c'est le seul qui tente vraiment de lier la forme cinématographique à la forme de la ville. D’abord furtive, vive, énervée, voire intrépide et risquée, la conduite manifeste le caractère foncièrement individualiste du personnage. Le regard cinématographique qui lui est lié suggère que le conducteur, pourtant photographe, ne s'intéresse pas aux espaces qu'il traverse. Le cinéaste, feignant aussi de ne pas s'y intéresser, a bien soin pourtant de filmer la rue, la furtivité du filmage donnant une forme à son regard. De façon à ce que l'on puisse observer de quoi elle est faite : des bâtiments bas et peints en rouge, une couleur qui, si elle peut faire couleur locale, n'en a pas moins une finalité cinématographique: servir de contrepoint à un élément architectural à venir, la maison bleue. Il n'est d'ailleurs pas impossible de penser qu'Antonioni ait choisi cette rue pour sa couleur vive et fraîchement peinte ${ }^{6}$. Le point de vue est d'abord par-dessus l'épaule du conducteur puis 
près de lui, sur le siège, en contre-plongée (plus une fois depuis le siège arrière en contre-plongée) : nous sommes bien dans la voiture et dans le film. C'est le point de vue d'un passager clandestin, un tiers fantomatique présent dans tout le film, manifesté souvent par le regard de la caméra $^{7}$, ce qui est une caractéristique du cinéma d'Antonioni. L'acteur, lui, garde les yeux sur son parcours, droit devant lui, vers son destin. On peut dire qu'il est alors paradoxalement fondu dans la ville, puisque la traversant sans la voir, il ne s'en exclut pas en se marquant dans une position de regardeur qui nécessiterait un recul, comme il aura d'autres occasions de le faire ensuite... ${ }^{8}$ Il faut s'intéresser à la versatilité du point de vue, d'abord dans le dos, puis à côté du conducteur, puis de nouveau dans le dos vers la fin de la séquence. En effet, un point de vue unique n'aurait proposé qu'une vision restreinte de la ville et du trajet. Ici au contraire, cette vision dynamique, changeante mais pas aléatoire, est totalement conditionnée par trois paramètres précis :

14 - la nécessité filmique de rendre compte cinématographiquement de la traversée ;

15 - l'environnement lui-même et sa configuration variable ;

16 - la clandestinité du regard qui est une constante du film : on n'y sait jamais vraiment qui représente le point de vue de la caméra, le cinéaste, le protagoniste ou encore, comme je l'ai dit à l'instant, un "tiers qui marche à [ses] côtés " pour reprendre l'expression de TS Elliot, et qui épaissit le mystère du film'.

17 Cette versatilité du point de vue, le film en joue sans cesse, mais ici elle dispense très précisément un "point de vue ", ou plus exactement un sentiment particulier que l'on pourrait traduire par l'expression : « curiosité manifeste ${ }^{10}$. Rappelons pour l'anecdote, les propos du peintre Georges Braque à propos de la vision perspectiviste conventionnelle: : [...] le point de vue est une toute petite chose. C'est comme celui qui toute sa vie dessinerait des profils en faisant croire que l'homme n'a qu'un seul œil ${ }^{11}$. Certes minime mais efficace, la variation du point de vue mobilis in mobile propose ici trois types de vision que le cinéaste tente de mettre en crise pareillement:

18 1. la vue perspectiviste illusionniste depuis l'arrière de la voiture vers l'avant, prenant la rue en perspective, mais saisissant dans son champ des objets l'empêchant de «bien voir ", ou des objets bougeant de façon à en perturber l'appréhension (la camionnette noire) ;

19 2. la mobilité de ce point de vue perspectiviste dans le dispositif de la caméra embarquée : le cabriolet, conduit de façon pour le moins sportive propose ainsi une incessante remise en cause de la vision dans le «ballet » automobile ;

20 3. enfin, la vue de côté en contreplongée qui fait semblant de s'intéresser au profil du conducteur mais perturbe la vision du spectateur par le défilement incessant des maisons rouges en arrière-plan. Ne dit-on pas, dans les vieux manuels de peinture, que "le rouge avance", d'un point de vue perceptif, et que cette couleur aura donc tendance à venir au regard en premier, à rendre ambigu l'espace, en creusant l'espace à rebours vers le spectateur et en ramenant le fond vers l'avant, au risque d'occulter le premier plan...

21 Puis le rythme de la séquence devient plus lent, nous arrivons dans un quartier hors de la zone "naturelle» du photographe, un autre Londres émergeant dans une architecture en construction au milieu d'un réseau urbain vieillot - petits bourgeois contre prolétaires... Ce nouveau rythme suggère une attention nouvelle, mais peut-on la prêter vraiment au conducteur? En effet, ne connaissant pas ce quartier, le 
photographe pourrait y manifester plus d'intérêt en tant que professionnel du regard. Mais lorsque la caméra panoramique vers le paysage urbain, une fois passée la maison bleue, le regard de la caméra, très attentif cette fois-ci, reste par-dessus l'épaule du photographe. C'est toujours le couple cabriolet-caméra qui est à l'œuvre. Le film, ici, nous offre un étonnant moment de mise en scène urbaine et cinématographique. La figure de la maison bleue comme aboutissement et frontière de la ville «traditionnelle » est caractéristique. Du point de vue perceptif, après la violence du rouge irritant des plans précédents, elle apaise la séquence autant que la rupture de rythme du montage. Ensuite, elle fonctionne comme un octroi symbolique, un check point plastique entre un domaine où le photographe est maître de ses images et un territoire encore inconnu où le sujet du film peut enfin démarrer, dans une altérité totale des images, des personnes, des lieux enfin. Au bout de la rue, nous trouverons ainsi le magasin d'antiquités, sorte d'aleph borgésien où s'agglomèrent dans un bric-àbrac visuel et matériel les espaces et les temps, l'ancien et nouveau, le grec et le chinois, le classique et le romantique, le chic et le kitsch.

La présence forte de la maison bleue n'est sensible que par le ralentissement du rythme de la voiture comme du film et le panoramique embarqué qui, tout en glissant lentement sur elle, insiste sur sa présence. La maison elle-même est une sorte d'avertissement pour dire que quelque chose change ou va changer - figure narrative à laquelle Antonioni est habitué. Sa singularité vis-à-vis des maisons rouges précédentes signale un monde décalé, étrange, mystérieux. Il s'agit dans cette séquence de payer un tribut à la vision de la ville, et d'accéder à un autre monde, littéralement, pour lequel il est également besoin de "passer le pont " (la maison bleue) pour voir les fantômes apparaître ${ }^{12}$, comme dans le Nosferatu de Murnau. Et, effectivement, le paysage urbain change de façon radicale après cet «octroi » visuel, et le couloir de brique de la rue londonienne fait place à un espace plus ouvert, des habitats collectifs modernes en construction, presque les mêmes avec leurs variantes plastiques que ceux qui initient L'Avventura... Et Antonioni prend son temps pour nous les montrer, même si les deux plans qui inspectent ce nouvel environnement sont relativement courts.

J'ai dit «fantômes » : est-ce seulement une figure de plus, dans l'esprit du temps, qui viendrait appuyer la question de la mort qui va apparaître plus tard dans le film? Notons que, dans le plan qui fait lien entre les deux univers urbains, tout de suite après la maison bleue apparait une cheminée d'usine, puis ces bâtiments en construction, dans un curieux renversement que le mouvement de la caméra rend seul possible. En effet, la cheminée est plus loin dans l'espace mais apparaît à l'écran avant les nouveaux immeubles en chantier. Cette présentation fait sens, elle indique un ordre dans la chaîne industrielle ou encore le remplacement d'un monde par un autre, à la fois relégué (son éloignement spatial) et remplacé (sa démolition probable pour faire place aux immeubles) ${ }^{13}$. Mais au-delà de cette interprétation somme toute aléatoire, la cheminée et la maison bleue restent deux "signes » urbains forts qui sursignifient le nouveau monde que l'on pénètre à l'instant et dont le mystère va très vite s'incruster dans l'image au plan suivant, sans que personne encore, ni le spectateur du film ni le personnage de la fiction, ne puisse l'identifier, cette enseigne de néon que l'on reverra dans la suite du film et qui y jouera un rôle ambivalent dont l'une des dimensions reste l'expression de l'étrangeté de l'incompréhension du monde ${ }^{14}$. 


\section{Passages, traversées} est doublé d'un travail de la focale qu'il faut ici noter. Si dans la plupart des plans la caméra est immobile, deux ou trois usent d'une mobilité double: d'une part, une " agilité » de la caméra portée visant à produire une image alerte et mouvante, et d'autre part, un usage nerveux du zoom avant et arrière portant le regard sur des éléments urbains qui, parce qu'ils sont pris dans la rapidité du plan et du mouvement, ne sont pas là pour être vus mais pour être l'image furtive du regard. Au final, on peut sentir une certaine insignifiance (au sens propre du terme) de ce territoire de passage, cet « entre-lieux » trouble, flou, bougé qui n'offre pas d'image certaine, construite avec la précision d'un dessin d'architecture ou d'une photographie documentaire. Mouvement, déplacement : c'est la caméra qui bouge, mais qui fait aussi bouger par son propre mouvement la fixité supposée de la ville, dans une figuralité paradoxale. De quel espace urbain, sinon un univers visuel et spécifique, Antonioni dresse le portrait de la même façon que, avec des moyens différents, Turner dans ses croquis de voyage fait depuis la diligence ou le coche d'eau ${ }^{15}$ ? De quoi peut bien rendre compte cette cinématographie-là, toute de versatilité plastique, sinon d'une ville que l'on perçoit entre deux plans à la fois diverse et en mouvement?

Tout Blowup n'est qu'une succession d'hétérotopies constituées en " cercles infernaux » concentriques autour du lieu central (le laboratoire) : la maison de Bill, le peintre, l'asile de nuit, le parc, le magasin d'antiquités, la salle de concert, le restaurant, la villa bourgeoise... Chacun de ces lieux possède à son tour une structure divisée en lieux spécifiques marqués d'une très forte singularité fonctionnelle ou symbolique. Mais, différent de celui que l'on quitte et de celui où l'on va, ce lieu entre les lieux que constitue la traversée propose une forme singulière dans sa différence avec les lieux circonscrits, repérés, définis architecturalement ou urbanistiquement. Il peut être la synthèse ou la recomposition prismatique ou kaléidoscopique de la ville. Et, du point de vue de l'imaginaire, comme on ne connaît pas celui où l'on va et qui se révèlera le lieu hétérotopique par excellence, l'entre-lieu peut-il en constituer les prémices? Enfin, est-il lui-même une forme nouvelle d'hétérotopie, qui pourrait être en toute occasion nommée la Traversée, si tant est que celle-ci, dans diverses pensées symboliques, est effectivement relative à l'interprétation symbolique de la mort ? Et si je propose cela, c'est aussi bien parce que l'hétérotopie souveraine est bien, pour Michel Foucault ${ }^{16}$, le cimetière, et que dans la suite du film, le déplacement se conclura dans un parc où se trouve un cadavre, le parc lui-même - sans croire qu'Antonioni ait pu le choisir à cause de cela - s'étant appelé dans l'histoire Hanging Wood avant de devenir Maryon Wilson Park, de ce fait lieu probable d'exécutions capitales ${ }^{17}$... De bien troublantes coïncidences qui font le sel des dérives interprétatives, mais il faut bien admettre aussi que les cimetières font partie de la ville. Il s'agit peut-être d'un autre sujet, différent de celui qui nous occupe et qui reste la traversée des villes, mais il lui est lié si l'on veut comprendre ce qui se joue dans l'interprétation de ces figures du passage. 
27 Passer d'un lieu à un autre, c'est passer à travers l'espace qui les relie. Nous postulons ici que cet espace de liaison est aussi un lieu que le cinéma construit. Qu'est-ce qu'un lieu et qu'est-ce qui le différencie de la notion abstraite d'espace ? C'est le fait d'être justement traversé, dans l'histoire, dans la culture, mais aussi dans la sensibilité, c'est-àdire dans l'actualité et le corps, par des actions qui le créent comme territoire d'expériences, ici comme expériences audio-visuelles. Et c'est bien l'action qui crée l'hétérotopie, la met à jour, la révèle et la rend possible, la sort de l'inconnu. Le cimetière de Foucault naît de la conjonction de la mort et du rite, de la croyance et de la nécessité, pas seulement de sa radicale différence avec les autres lieux. Pour construire cet autre lieu, le cinéma propose au moins deux solutions :

- l'ellipse (via le montage cut), qui est une éclipse de temps ;

- le fondu (via la surimpression) qui est un passage fusionnant les temporalités.

La traversée de Blowup n'est pas un fondu et n'est d'ailleurs pas dépourvue d'ellipse, mais elle détaille et caractérise cette ellipse, elle la distend: elle est à la fois temps (montage) et espace, cette conjonction qualifiant précisément le lieu dans une durée et comme conflagration de lieux. Dans Blowup, les liaisons sont toujours soit des ellipses ${ }^{18}$, soit des crises de la représentation dans une fausse continuité (ruptures, montage, formes plastiques): à ce moment ce sont des espaces hétérotopiques, ou des "glissières" spatiotemporelles sur lesquelles se positionnent des arrêts, des "stations », des sortes de pycnoses permettant l'irruption du " hasard », d'accidents de parcours qui vont justement changer le cours des choses ou ne servir à rien, sinon à donner du sens à moudre à la représentation générale du monde construite par le film, comme par exemple la séquence du concert des Yardbirds. Ainsi encore, au retour du parc, le photographe glisse le long des rues et croise une manifestation anti-nucléaire : outre l'aspect « esprit du temps » qu'elle porte aujourd'hui, elle n'a d'autre utilité, dans le sentiment d'arrêt ou de distraction qu'elle provoque, que de placer un élément d'intrigue quasi invisible, et qui renforce l'idée d'une filature. Dans l'ensemble du film, et pas seulement dans la scène qui nous occupe plus précisément (l'aller au parc), le sentiment d'hétérotopie se manifeste par des effets cinéplastiques et sonores: alternance silence/bruit (dans la séquence d'aller au parc, puis le parc lui-même), le vent, la confrontation nature/culture, les formes différentes des espaces qui se succèdent, les points de vue variés (subjectifs/non subjectifs/embarqués, etc.), et la singularité des objets (la tour lumineuse, la maison bleue) et des êtres (les deux homosexuels et leur caniche, le vieux vendeur de la boutique, les manifestants dont certains sont caractérisés). Au bout du compte, si la traversée se voit comme une autre sorte d'hétérotopie, c'est aussi qu'elle se construit sur des traces, des ébauches de lieux furtifs.

\section{Un lieu radical : la traversée de Los Angeles dans Zabriskie Point}

31 Si dans Blowup on traverse la ville pour aller d'un endroit à un autre, et cela à plusieurs moments du film, avec Zabriskie Point on traverse pour traverser : la traversée est le lieu même du film - de presque tout le film d'ailleurs, en tant que road-movie. Cette séquence de la traversée de L.A. en pick-up constitue un entre-lieu pour lui-même sans qu'aucune nécessité diégétique ne soit manifeste. L'ellipse aurait suffi, puisque par 
ailleurs, Los Angeles est une ville montrée ici sans formes, sans « lieux » véritables, et constituée par le film comme une succession de points de vue très focalisés, de détails qui peinent à former un tout : aussi nous n'allons pas d'un endroit spécifique à un autre mais nous nous déplaçons dans un monde toujours identique à lui-même, comme dans un jeu vidéo, par exemple... Le film lui-même, au moins pour tout ce qui se déroule à L.A., est construit non pas au hasard mais selon l'idée d'une non-relation entre les séquences, que le récit relie à peine. Une inversion entre elles pourrait se faire, on pourrait mélanger les bobines... ${ }^{19}$ Non, gageons simplement que l'«utilité » de la séquence, si besoin est, se tient dans ce penchant documentaire qui irrigue incessamment le cinéma d'Antonioni et qui n'était pas absent des images de Londres : il s'agit ici de nous montrer la vision que le cinéaste s'est construit de cette ville, précisément. Ou encore, on pourrait se demander si cette traversée de Los Angeles qui nous occupe ici n'est pas aussi un film dans le film, un autre film ou le germe d'un autre film (expérimental par exemple), tant il se démarque esthétiquement du reste.

De quoi cette séquence est-elle faite, précisément? La séquence démarre sans que l'on puisse dire où vont les protagonistes, ils se déplacent simplement, ils traversent un espace qui ne change pas vraiment tout au long de la séquence. Par contre, le film, lui, change : la séquence se singularise très fortement dans l'ensemble du film; la séquence est faite de moments malgré tout différents, fondés sur la vision de la voiture-caméra. On peut considérer que son point de départ est d'ailleurs un point de vue très particulier qui manifeste l'attrait d'Antonioni pour la présence d'images fixes dans tous ses films. En trois ou quatre plans, le cinéaste « photographie » une grande peinture murale, bien connue à l'époque ${ }^{20}$, représentant avec humour et peut-être un peu de nostalgie le passé rural des USA sur lequel s'est en partie fondé autant le mouvement hippie, une partie de l'art américain que les pensées les plus réactionnaires. Ce déplacement pseudo temporel inaugure-t-il $d u$ déplacement spatial, très problématique, que la séquence opère ? En tout cas, on peut dire aussi qu'il produit un anachronisme ou un glissement qui intègre ironiquement la camionnette dans ce décor dont elle n'est finalement pas exclue de par sa relative ancienneté, avec un relent visuel style Raisins de la colère... Cet "anachronisme » ou plutôt ce décalage entre l'époque réelle et l'automobile, le film le cultive aussi avec l'autre automobile, celle conduite par la fille et qui, effectivement, est une voiture ancienne dans son style années 1950 très marqué21. Bien entendu, ce ne sont pas des Rolls comme dans Blowup, et cela marque aussi sociologiquement les personnages, mais c'est pourtant ce décalage-là qui accompagne le décalage global du film. Le passé s'incruste dans le présent de la ville qui semble ne pas avoir lieu, pour jouer sur l'ambiguïté de l'expression. Ainsi lorsque la camionnette apparait dans le champ du panoramique qui balaie la fresque, l'ensemble forme un drôle de paysage composite qui, un instant, nous transporte dans les années 1950, voire au-delà, dans une traversée du temps dont il faudra attendre la fin de la séquence pour découvrir brutalement que nous en sommes revenus. Tout a l'air désuet dans le temps réel.

Un dernier coup d'œil à la peinture depuis le pick-up et nous voilà maintenant embarqué, à moitié observateur et à moitié observant : je ferai ici cette distinction entre les plans filmant de l'extérieur la cabine du véhicule (observateur), et ceux filmant la ville depuis l'intérieur de l'habitacle (observant), en fait depuis le plateau du pick-up au travers du pare-brise arrière. Il n'était pas possible d'opérer cette distinction dans Blowup, elle ne pouvait s'appliquer au « passager clandestin ». 
Une constante, passé le plan du doigt d'honneur aux deux motards : la focale longue de la caméra, quelle que soit sa position. Ce qui induit un regard focalisé sur les détails, excluant le contexte, ou le rendant flou, abstrait comme dans ce plan assez long dont nous ne pouvons décider s'il est fait d'un plan continu ou d'un montage de plans plus abstraits et rapides les uns que les autres. Ce point de vue exclusif et excluant nous laisse ainsi dans cette indécision quant au temps et à l'espace dans lequel nous sommes. Du dehors nous ne voyons que les passagers, et du dedans que les détails du paysage urbain, enseignes (qui semblent les mêmes depuis une époque plus ancienne), morceaux de bâtiments commerciaux et industriels, signalisation, camions... couleurs, formes sans signification précise. Le rythme rapide et saccadé associé à des mouvements brusques de la caméra vient à n'en pas douter de la séquence de Blowup que nous avons abordée, mais celle-ci était en son naturel. Ici, fait très notable et presque nouveau chez Antonioni, la séquence est accompagnée d'une bande son que ne dénierait pas un groupe de rock punk industriel ${ }^{22}$. Et cela jusqu'à l'apaisement de l'avenue bordée de palmiers où la séquence se transforme - un peu comme après la maison bleue de Blowup - en un rythme plus paisible, avec cette fois-ci la légèreté de l'épisode de rencontre avec la sœur du héros... Ce moment ne marque pas seulement un changement dans le temps (durée et rythme) mais aussi dans l'espace puisque d'une certaine manière, en passant des enseignes et des usines aux palmiers, nous changeons d'univers...

Ce portrait de ville n'est pas sur le principe si différent de celui de Londres. Mais là où malgré tout Antonioni opérait avec des focales plus larges, nécessitées par la densité de l'architecture, il use ici d'effets de zoom très brusques, d'une focale longue brisant l'effet de profondeur, aplatissant l'image et rendant difficile la perception d'un espace, bref : la séquence forme de la ville une image plate, plate comme la fresque du début qu'Antonioni ne regarde jamais de face comme un trompe-l'œil, mais bien comme une image-objet, inscrite dans la réalité, donc possiblement visible de biais par exemple et déformée selon le point de vue. Là est sans doute la vraie raison cinématographique - et non accessoire ou couleur locale - de la présence de la fresque en début de séquence. En fin de compte, il semble impossible, arrivé au bout de la séquence, de reconstituer ne serait-ce qu'une image hiérarchisée d'un espace urbain dont nous n'avons traversé que des détails : nous restons dans une mosaïque, ou plus encore dans un kaléidoscope où les espaces sont identiques et arbitrairement associés.

\section{Pour (ne pas) conclure}

Par cette intervention, j'ai souhaité penser la représentation de l'espace urbain dans le filtre du cinéma, en prenant le cas particulier d'un cinéaste que je connais bien. Deux ou trois choses me semblent nécessaires à remarquer à la fin de cette analyse.

- Antonioni n'est évidemment pas le seul cinéaste à s'intéresser, même en passant, à l'urbanité. Nombreux sont les cinéastes qui interrogent le rapport des hommes et des femmes à ce milieu qui est notre monde ordinaire, et qui est le lieu même du cinéma. Un festival (au moins) leur est consacrée ${ }^{3}$. En français, une encyclopédie ${ }^{24}$ et de nombreux ouvrages. Des premiers films des frères Lumières aux mondes divers d'Abbas Kiarostami, Johan Van der Keuken, Raphaël Nadjari, Amos Gitaï ou Jia Zhang Ke, le cinéma contemporain a repris ce flambeau porté par les générations successives de réalisateurs, tant dans le champ du documentaire que de la fiction, et quels que soient 
les genres, y compris le film d'animation, le fantastique, la science-fiction (et je dirais même surtout, puisque toujours elle a eu le privilège de poser en termes créatifs et pointus ces questions urbaines que sont l'aliénation, la forme, la vie en commun : Blade Runner ou Soylent Green en témoignent). Mais alors pourquoi Antonioni? J'aurais tendance à répondre par une esquive : c'est celui que je connais le mieux... mais c'est aussi celui qui, dans les années où je me suis formé à l'art et au cinéma, m'a le plus fourni l'envie de le connaître, à cause du mystère qu'il construisait dans ses films; de même qu'il m'est apparu comme le plus à même d'expliquer, par les moyens propres à son art, par la sensibilité et l'imaginaire, le fond de l'aliénation qui nous lie à ce milieu.

- J'en viens donc à l'autre remarque qui découle de la précédente. Antonioni c'est aussi l'époque. Les années 1950, 1960 et un peu au-delà. Ces années-là, ces années "pop ", ont été celles d'une immense ouverture de l'art - et sans doute d'autres champs de l'imaginaire et du sensible - à laquelle nos générations ont voulu participer : cette participation a marqué évidemment la suite des événements, culturels, artistiques, politiques. Inutile de les rappeler, mais cela a enclenché une habitude, celle que la jeunesse a gardé depuis; se mêler de ce qui la regarde, et au plus haut point le monde dans lequel elle est propulsée brutalement, et l'art qui en tient le compte précis à l'aide de moyens spécifiques. Mais par-delà ces considérations un peu générales et peut-être narcissiques et nostalgiques que vous me pardonnerez, il faut dire qu'Antonioni est l'un de ceux qui a le mieux marqué cette époque en ce sens, sans le dogmatisme trop souvent évoqué quand on s'en prend à cette époque. Antonioni fait époque, si vous me permettez de le dire ainsi, non pas dans le sens commun et opportuniste où son art serait empreint des caractères des années 1960, mais à l'inverse en ce que son cinéma a marqué irrémédiablement la construction esthétique et sensible de cette époque, en rendant visible l'aliénation et l'inadaptation dans lesquelles le capitalisme nous a alors plongé. Lorsque, dans L'Éclipse, Antonioni montre Monica Vitti cherchant à placer dans son intérieur une plaque minérale contenant le fossile d'une plante, la séquence en dit long de cette incapacité à trouver la correspondance entre le monde contemporain et notre vie, entre les profondeurs que nous habitons et la surface qui nous supporte.

- Si ces films sont ceux d'une époque, ils seraient donc également éphémères... Mais qu'en est-il vraiment? Ne sont-ils que des témoins désormais éloignés de l'ambiance de ces années, Blowup n'est-il à regarder que comme un reflet nostalgique du swinging London et des années pop ? Dans ce cas, sa permanence dans notre imaginaire ne seraitelle qu'un effet de cette nostalgie? J'en doute, bien que personne ne soit à l'abri d'une telle réaction... Mais cette prégnance, qui est plus qu'un écho, ne montre-t-elle pas justement qu'au-delà de l'éphémère il reste justement une empreinte dont nous savons aussi qu'elle nous a formé pour les temps qui sont venus après, et qu'elle a été une matrice de sensibilité et d'imaginaire, peut-être de pensée, pour la suite de nos parcours artistiques et intellectuels. Un effet-mère, plutôt. 


\section{BIBLIOGRAPHIE}

Benjamin Walter, Paris, capitale du XIX ${ }^{\mathrm{e}}$ siècle, Jean Lacoste (trad.), Paris, Éditions du Cerf, 1989.

Deleuze Gilles, L'image-mouvement, Paris, Minuit (Cinéma 1), 1983.

Dora Vallier, « Braque, la peinture et nous », Cahiers d'art, $29^{\mathrm{e}}$ année, $\mathrm{n}^{\circ}$ 1, Paris, octobre 1954.

Foucault Michel, « Des espaces autres », in Dits et écrits, vol. II 1976-1988, Paris, Gallimard, 1994.

Greenberg David, Smith Kathryn, Teacher Stuart, Big Art - Megamurals and supergraphics,

Philadelphia, Running Press, 1977.

Jousse Thierry, Pacquot Thierry (dir.), La ville au cinéma : encyclopédie, Paris, Cahiers du cinéma, 2005.

Moure José, Du vide au cinéma, thèse de doctorat.

Robic Jean-François, Blowup, une seconde vue, Paris, L'Harmattan, 2012.

Virilio Paul, Esthétique de la disparition, Paris, Galilée, 1989.

Žižek Slavoj, Lacrimae rerum : cinq essais sur Kieślowski, Hitchcock, Tarkovski et Lynch, Paris,

Éd. Amsterdam, 2005.

Filmographie :

Nosferatu, Friedrich-Wilhelm Murnau, 1922.

La Croisière du Navigator, Buster Keaton, Donald Crisp, 1924.

Les Gens du Po, Michelangelo Antonioni, 1943.

Nettoyage urbain, Michelangelo Antonioni, 1948.

L'Avventura, Michelangelo Antonioni, 1960.

L'Éclipse, Michelangelo Antonioni, 1962.

Désert rouge, Michelangelo Antonioni, 1964.

Blow-Up, Michelangelo Antonioni, 1966.

Easy Rider, Dennis Hopper, 1969.

Zabriskie Point, Michelangelo Antonioni, 1970.

THX 1138, George Lucas, 1971.

Chung Kuo, La Chine, Michelangelo Antonioni, 1972.

Profession : reporter, Michelangelo Antonioni, 1975.

Lo sguardo di Michelangelo, Michelangelo Antonioni, 2004.

\section{NOTES}

1. Walter Benjamin, Paris, capitale du XIX $x^{e}$ siècle, Jean Lacoste (trad.), Paris, Éditions du Cerf, 1989.

2. Voir Les Gens du Po en 1943 et Nettoyage urbain (NU) en 1948, entre autres... Souvenons-nous également de Chung Kuo, La Chine en 1972. Son dernier film, Lo sguardo di Michelangelo (Le Regard de 
Michelangelo), peut être considéré comme tel, malgré l'aspect personnel du regard, justement : il boucle la boucle esthétique de la carrière de l'artiste.

3. Paul Virilio, Esthétique de la disparition, Paris, Galilée, 1989.

4. Et si la voiture avec son pare-brise est une salle de cinéma, alors la décapotable c'est le Kinopanorama! Les années 1960 sont l'époque d'un cinéma toujours plus large (la hauteur est une caractéristique niée par le cinéma). Les trois écrans du Kinopanorama sont le début de tous les hyper-cinémas qui aboutissent à la 3D actuelle.

5. On se souvient du personnage incarné par Keaton dans La Croisière du Navigator, lorsqu'il se fait conduire en décapotable depuis son domicile chez sa fiancée Betsy O'Brien, de l'autre côté de la rue. La voiture ne fait qu'un demi-tour d'un trottoir à l'autre. Ici, la voiture ne sert qu'à l'ostentation de la richesse et de la paresse sociale qu'engendre la fortune, et le regard que le cinéaste porte sur la ville est délié de la voiture.

6. En pensant notamment à la manière dont il a fait repeindre une rue de Ravenne pour Le Désert rouge. Plus difficile à faire dans un Londres hyperactif, il aurait bien pu profiter d'un ravalement récent.

7. Idée développée dans mon ouvrage Blowup, une seconde vue, Paris, L'Harmattan, 2012, p. 63. Voir également Slavoj Žižek, Lacrimae rerum : cinq essais sur Kieślowski, Hitchcock, Tarkovski et Lynch, Paris, Éd. Amsterdam, 2005.

8. Cette position de passager est reprise dans Profession Reporter dont le titre international, rappelons-le, est justement The Passenger.

9. Voir aussi Slavoj Žižek, Lacrimae rerum : cinq essais sur Kieślowski, Hitchcock, Tarkovski et Lynch, Paris, Éd. Amsterdam, 2005 qui explore cette question pour Hitchcock, ainsi que pour Antonioni : Gilles Deleuze, L'image-mouvement, Paris, Minuit (Cinéma 1), 1983, et José Moure, Du vide au cinéma, thèse de doctorat.

10. Et certainement pas d'« inquiétante étrangeté » qui, si elle peut apparaître ailleurs dans Blowup, n'est pas en jeu ici, sinon dans la reprise du sentiment dans une traduction plus juste de son « appellation d'origine », une inquiétante familiarité...

11. Entretien avec Dora Vallier, in Dora Vallier, « Braque, la peinture et nous ", Cahiers d'art, $29^{\mathrm{e}}$ année, $\mathrm{n}^{\circ} 1$, Paris, octobre 1954, p. 14.

12. Voir le cartel de Murnau, Nosferatu, «Et quand il eut dépassé le pont, les fantômes vinrent à sa rencontre ». Le cartel français est simple et frappant, la version allemande est plus longue et « littéraire ». Il y a dans le film de Murnau un marqueur visuel dans le passage de la pellicule au négatif, comme il en existe un dans Blowup avec la maison bleue.

13. En consultant Google Maps, il est difficile de reconnaître le quartier... Tamar Street, au coin de laquelle se tenait la boutique, est devenue un passage privatif et toute la continuité de la rue en amont a fait place à des immeubles et des voies rapides... La maison bleue et la cheminée ont apparemment disparu, même s'il existe encore des bâtiments industriels aux environs...

14. On sait que cet emblème de néon a été placé là par Antonioni, et que le signe ne signifie rien, ne renvoie à rien, que malgré les apparences, ces sortes de ruines modernes ne sont donc pas des mots compréhensibles, n'ont pas de sens lisible.

15. Au demeurant, sans doute invente-t-il un dispositif dessin-diligence équivalent à celui qui nous occupe?

16. Michel Foucault, «Des espaces autres », 1967, prononcé au Cercle d'études architecturales et publié la première fois en 1984, dans Architecture, Mouvement, Continuité, $\mathrm{n}^{\circ}$ 5, octobre 1984. In Dits et écrits, vol. II 1976-1988, Paris, Gallimard, 1994. Le décalage de date entre la conférence et sa publication s'explique par le fait que Foucault n'a autorisé sa publication qu'en 1984.

17. Bien qu'il existe d'autres étymologies.

18. Il faut bien réduire les 24 heures de l'histoire dans les 112 minutes du film...

19. Je me souviens d'avoir vu au cinéma Le Styx, en 1971, le film de George Lucas, THX 1138, les bobines ayant été inversées... et je crois bien ne m'en être pas rendu compte. Il y a des films 
comme ça... C'est peut-être un gage de qualité ? Ou le symptôme d'une époque, les deux films, Zabriskie Point et THX 1138, n'ayant que quelques mois de différences. Rappelons-nous par ailleurs qu'Antonioni tournait son film en même temps que Dennis Hopper tournait Easy Rider au même endroit (Daria Halprin, actrice de Zabriskie Point, allant devenir l'épouse de D. Hopper). Comme beaucoup de road-movies, celui-là également est constitué de nombreuses scènes invariablement équivalentes et interchangeables.

20. La peinture représentée est Hog Heaven (le paradis des cochons) de Les Grimes et Arno Jordan, réalisée de 1957 jusqu'à la fin des années 1970. Les Grimes, à l'origine peintre de décors pour les studios de cinéma, s'est tué en 1968, en tombant de l'échafaudage pendant qu'il peignait un morceau de ciel de cette peinture murale. Jordan a pris sa succession pour finir cette œuvre. Voir David Greenberg, Kathryn Smith, Stuart Teacher, Big Art-Megamurals and supergraphics, Philadelphia, Running Press, 1977. (Note prélevée de J.-F. Robic, Blowup, une seconde vue, Paris, L'Harmattan, 2012).

21. Buick spéciale De Luxe 48-D modèle 1951. Merci à Jean-Louis Hess, qui m'a aidé à retrouver ce modèle. Voir aussi le site internet http://www.imcdb.org/

22. On peut rapprocher cette musique du générique du Désert rouge, et plus encore de la séquence finale de L'Éclipse, affectée du même genre de musique. Mais l'usage de musique off ne commence vraiment chez le cinéaste qu'avec Zabriskie Point, et encore toujours dans un jeu subtil entre in et off : voir J.-F. Robic, Blowup, une seconde vue, p. 515.

23. Festival Ville et cinéma : Métropoles, Paris, 2012.

24. Thierry Jousse, Thierry Pacquot (dir.), La ville au cinéma: encyclopédie, Paris, Cahiers du cinéma, 2005.

\section{RÉSUMÉS}

Le cinéma constitue l'un des moyens de percevoir l'espace urbain de manière spécifique, notamment par les déplacements au travers des villes - tout en (se) jouant de ses effets propres : montage, temporalité, espace de l'image. Les films d'Antonioni servent bien ce propos au travers de dispositifs particuliers de mise en scène et de filmage où la voiture alliée à la caméra fait office de dispositif perceptif pertinent. Dans L'Avventura et surtout dans Blowup et Zabriskie Point, ce dispositif érige le déplacement en quasi hétérotopie tant il permet de concevoir cet entre-lieu à la fois spatial et temporel comme un espace-temps spécifique où l'image de la ville se projette et se recompose de manière poétique et mystérieuse, pleine de potentialité esthétique et sémantique.

\section{INDEX}

Mots-clés : cinéma, Antonioni, voiture, dispositif, médium, ville, traversée, hétérotopie 
AUTEUR

JEAN-FRANÇOIS ROBIC

Artiste, professeur en arts plastiquesUniversité de Picardie-Jules Verne 